\title{
Strong Birefringence Tuning of Optical Waveguides With Femtosecond Laser Irradiation of Bulk Fused Silica and Single Mode Fibers
}

\author{
Luís A. Fernandes, Member, OSA, Jason R. Grenier, Member, OSA, Paulo V. S. Marques, \\ J. Stewart Aitchison, Fellow, OSA, and Peter R. Herman, Fellow, OSA
}

\begin{abstract}
Birefringence tunability is demonstrated in waveguides formed in bulk fused silica and in the core of single mode fibers, by femtosecond laser writing of stress inducing tracks that are placed with different geometries around the core of the waveguides. The femtosecond laser generated stress effect was probed by the birefringence induced spectral splitting of either Bragg grating waveguides in bulk fused silica or weakly modulated, femtosecond laser induced Bragg gratings in optical fibers. Birefringence values as low as $4 \times 10^{-6}$ and up to $2 \times 10^{-3}$ were obtained by controlling the fabrication conditions such as the laser pulse energy, the writing femtosecond laser polarization, the number of overwriting exposures, and the geometry of the induced stress tracks. Wave retarders are developed and characterized by a cross polarization technique to provide the spectral response of the stress induced birefringence, offering the convenient fabrication of short length and broadband in-line polarization devices. With this approach, millimeter length tracks provided $10 \mathrm{~nm}$ bandwidth polarization retarders in a single mode fiber and $\mathbf{a} 65 \mathrm{~nm}$ bandwidth retarders in bulk fused silica.
\end{abstract}

Index Terms-Birefringent waveguides, fiber Bragg gratings, integrated optics devices, integrated wave retarders, laser induced birefringence, ultrafast laser materials processing.

\section{INTRODUCTION}

B IREFRINGENCE, defined as the difference between the effective refractive indices of the two polarization eigenmodes $(\Delta n)$, in waveguides and optical fibers is an important consideration in the design of optical circuits and fiber devices. Such birefringence may arise intrinsically from the microscopic structure of crystals, from the asymmetric geometry of waveguides, or may arise from stress rods in polarization maintaining fibers, thermal strain in multi-layer planar waveguides, or asym-

Manuscript received July 22, 2013; revised September 24, 2013; accepted September 25, 2013. Date of publication October 1, 2013; date of current version October 30, 2013. This work was supported in part by the Natural Sciences and Engineering Research Council of Canada and in part by the Canadian Institute for Photonic Innovations.

L. A. Fernandes, J. R. Grenier, J. S. Aitchison and P. R. Herman are with the Department of Electrical and Computer Engineering, Institute for Optical Sciences, University of Toronto, Toronto, ON M5S 3G4, Canada (e-mail: luis.nevespaivafernandes@utoronto.ca; j.grenier@utoronto.ca; stewart.aitchison@utoronto.ca; p.herman@utoronto.ca).

P. V. S. Marques is with the INESC-Porto, Departamento de Física e Astronomia da Universidade do Porto, Rua do Campo Alegre 687, 4169-007 Porto, Portugal (e-mail: psmarque@fc.up.pt).

Color versions of one or more of the figures in this paper are available online at http://ieeexplore.iee.org.

Digital Object Identifier 10.1109/JLT.2013.2284240 metric modifications from high intensity laser exposures [1]. Alternatively, femtosecond laser writing of stressing structures in the proximity of the waveguides and the femtosecond laser formation of nanogratings [2] to induce form birefringence are both attractive means for locally tuning waveguide birefringence and facilitating the formation of useful polarization components such as wave retarder or polarization beam splitters.

The degree of waveguide birefringence varies highly, from very low birefringence $\left(\approx 10^{-7}\right)$ in single mode fibers (SMFs) used in optical communications to minimize polarization mode dispersion and enable high speed optical data transmission, to high waveguide birefringence of $10^{-4}$ in polarization maintaining fibers, and can reach $10^{-2}$ [3] in microstructured fibers.

Polarization sensitive waveguide devices such as waveplates [4], polarizers, and polarization dependent directional couplers [5] can be fabricated with reduced sizes by increasing the birefringence. This is desirable in building compact optical circuits for polarization phase-shift keying in optical communication [6] and quantum optics applications [7], [8]. High waveguide birefringence of $\Delta n=2 \times 10^{-3}$ can manifest in large modal separation of $\approx 2 \mathrm{~nm}$ [9] in fiber Bragg gratings (FBGs), while also enabling new approaches in strain, pressure, and temperature sensing [3], [10], twist and torsion sensing [11] and single polarization operation of fiber lasers [12]-[14].

Local modification of the waveguide birefringence is desirable in the design of polarization responses in optical circuit components. To this end, femtosecond laser writing of stressing tracks has been shown [15] to generate a strong birefringence of $8 \times 10^{-4}$ when written around the core of a SMF while a value of $7.93 \times 10^{-4}$ was also found for a tenth order grating inscribed inside the fiber core [16]. Similar stressing tracks have also been used to tune the waveguide birefringence from $\approx 10^{-6}$ to $4.35 \times 10^{-4}$ [17] in waveguides written inside bulk silica glass with a femtosecond laser.

Femtosecond lasers offer the opportunity to develop new integrated photonic devices in optical fibers [18]. To develop this direction, the present paper extends the femtosecond laser stress tuning technique to high numerical aperture (HNA) lens focusing with oil immersion for the dual purposes of reducing optical aberrations at the glass-air interface and confining the laser interaction more tightly to increase the strength of the stress inducing tracks. Oil immersion further removes the distortions in the geometric placement of the laser modified track relative to the waveguides [19]. We report on femtosecond laser birefringence tuning of waveguides in both bulk fused silica glass and 


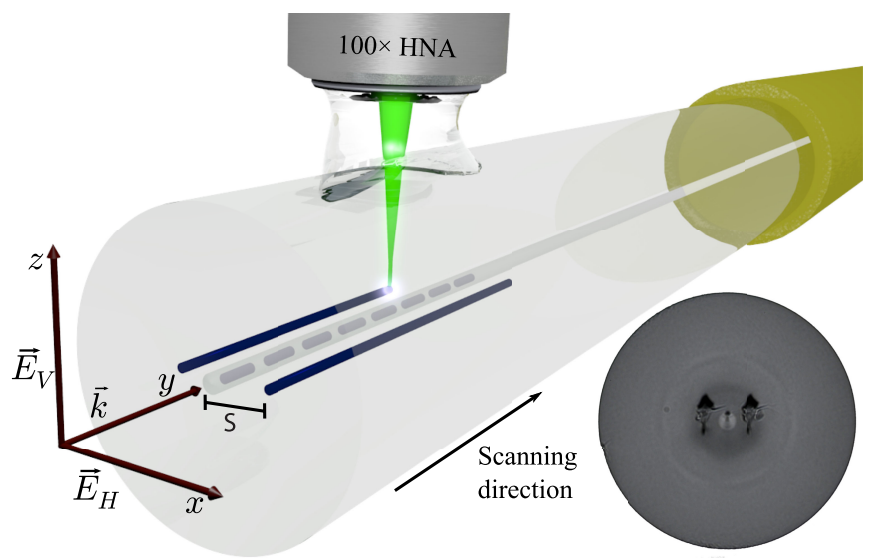

Fig. 1. Stress tracks are fabricated around Bragg grating waveguide (BGWs) in bulk glass and around the core of SMFs with inscribed FBGs. Vertical, $\vec{E}_{V}$, and horizontal, $\vec{E}_{H}$, are the orientations of the waveguide polarization eigenmodes, and $S$ is the center-to-center separation between the waveguide core and the stress inducing tracks. The picture in the bottom right corner shows an optical microscope image of a SMF with $125 \mu \mathrm{m}$ diameter cladding, a grating in the core, and a stress inducing track horizontally positioned on each side.

SMFs. The proximity, geometry, nanograting orientation, and overwriting of stress tracks near the waveguides were studied and optimized, yielding a record tuning range expanded more than two-fold, to a maximum birefringence of $\Delta n \approx 2 \times 10^{-3}$.

\section{DEVICE FABRICATION}

Stress inducing laser modified tracks were fabricated around waveguides in fused silica [17] and in the cladding of single mode optical fibers (SMFs) as shown in Fig. 1, using the second harmonic output of an Yb-doped chirped pulse amplified fiber laser (IMRA America $\mu$ Jewel D-400-VR) with a center wavelength of $522 \mathrm{~nm}$, a pulse duration of $\approx 200 \mathrm{fs}$, and repetition rates of $500 \mathrm{kHz}$ and $100 \mathrm{kHz}$. Waveguide and stress tracks were fabricated $70 \mu \mathrm{m}$ below the surface of bulk fused silica glass (Corning 7980) with either a 0.55 low numerical aperture (LNA) or an oil immersion $100 \times 1.25 \mathrm{HNA}$ lens, providing focal spot diameters $\left(1 / e^{2}\right)$ calculated to be $0.8 \mu \mathrm{m}$ and $0.4 \mu \mathrm{m}$, respectively. Alternatively, the laser focal position was aligned with better than $1 \mu \mathrm{m}$ accuracy to run parallel with the core of the SMF, over a length of $10 \mathrm{~cm}$, with an HNA lens.

Fused silica samples and optical fibers were mounted on an air-bearing motion stage (Aerotech ABL1000) with a resolution of $2.5 \mathrm{~nm}$, a repeatability of $200 \mathrm{~nm}$, and scanned at a constant speed of $0.268 \mathrm{~mm} / \mathrm{s}$ to define both waveguide and stress tracks. Bragg grating waveguides (BGWs) were also fabricated at the same time to independently check the waveguide propagation constants and birefringence [20], [21]. The BGWs are fabricated by introducing an acousto-optic modulator into the laser path to form burst trains [20] with modulation frequencies from $506 \mathrm{~Hz}$ to $496 \mathrm{~Hz}$ in order to obtain Bragg reflections from $1533 \mathrm{~nm}$ to $1565 \mathrm{~nm}$. The same technique was applied to accurately position weak Bragg gratings within the core of SMFs [19] to form FBGs. Polarization splitting of the reflection and transmission spectra [21] was measured to determine the stress induced birefringence generated by the surrounding laser fabricated tracks.

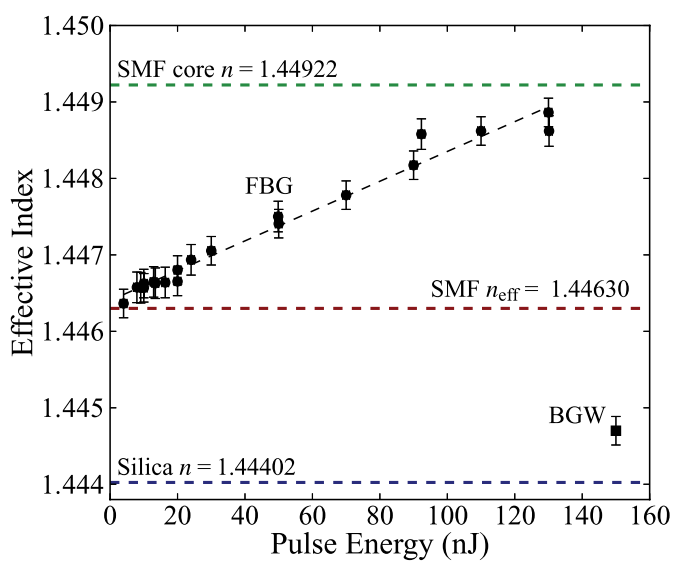

Fig. 2. Effective index of FBGs at $1550 \mathrm{~nm}$ wavelength as a function of the laser pulse energy (black circles) and the effective index of a BGW for a writing pulse energy of $150 \mathrm{~nJ}$ (black square). The values are compared with the standard index of refraction of the SMF core (green dashed line), fused silica fiber cladding (blue dashed line), and the effective index of the unmodified SMF mode (red dashed line).

The pulse energy used in the formation the stress inducing tracks was varied between $100 \mathrm{~nJ}$ and $420 \mathrm{~nJ}$ in order to examine saturation, maximum stress, and damage limits. The tunable range of the birefringence induced in SMFs and bulk fused silica was explored by varying the track position relative the waveguide under test and the separation, $S$, from $10 \mu \mathrm{m}$ to $70 \mu \mathrm{m}$ in fused silica and from $8 \mu \mathrm{m}$ to $45 \mu \mathrm{m}$ in SMFs. The stress tracks were positioned either horizontally or vertically with respect to the vertical writing laser direction, shown in Fig. 1, which shows an image of the fiber cross-section containing two parallel stress tracks written horizontally on either side of the core waveguide. The laser modification consists of zones of $8 \mu \mathrm{m} \times 20 \mu \mathrm{m}$ size. Multiple overlapping stress tracks were also applied to study the possibility of further birefringence increase and saturation.

The polarization of the writing laser was controlled to be parallel, $\vec{E}_{\mathrm{Par} \|}$, or perpendicular, $\vec{E}_{\mathrm{Per} \perp}$, with the waveguide direction in order to vary the nanograting orientation [22] and their strong form birefringence on modifying the waveguide core. Nanograting orientation in stressing tracks formed outside of the waveguide core was not previously found to contribute to a long reaching stress birefringence [17].

A pulse energy of $150 \mathrm{~nJ}$ was selected to write the BGWs in bulk glass, as found previously to offer low propagation loss of $\approx 0.5 \mathrm{~dB} / \mathrm{cm}[20]$. In order to study the influence of the femtosecond induced stress around the core of the SMFs, a grating needs to be inscribed in the fiber to facilitate the birefringence measurement. This process contributes a birefringence to the fiber mode. Fig. 2 presents a study of the effective index of the FBGs, compared with the effective index of the unmodified fiber (Corning SMF-28), as a function of the pulse energy. The effective indices $\left(n_{\text {eff }}\right)$ were calculated from the Bragg relation $\left(\lambda_{B}=2 n_{\text {eff }} \Lambda\right)$ given the known values of the periodicity $(\Lambda)$ and the measured Bragg resonance $\left(\lambda_{B}\right)$. Due to the higher photosensitivity of the germanium doped core relative to the fused silica cladding, a much smaller $(\approx 5 \mathrm{~nJ}$ to $10 \mathrm{~nJ})$ pulse energy was sufficient to inscribe weak FBGs in the SMFs and 


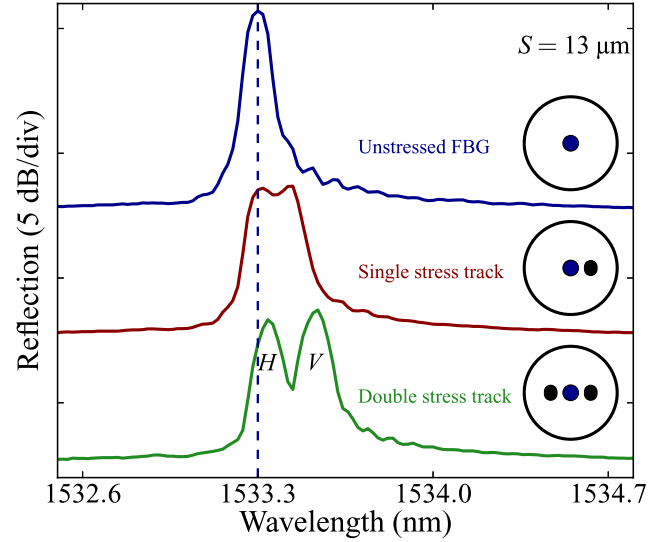

Fig. 3. Offsetted Bragg reflection spectra of a FBG where each stress track, symmetrically positioned in opposite sides of the SMF core, affects the birefringence cumulatively. The tracks are written with a separation $S=13 \mu \mathrm{m}$ and with $140 \mathrm{~nJ}$ of pulse energy alongside a $10 \mathrm{~mm}$ long FBG. The effective index of the $V$ mode increases significantly compared to the $H$ mode which is mostly unaffected.

thereby probe the birefringence induced solely by the stressing tracks with minimal effect on the propagating mode shape, the propagation loss, and the effective index from the femtosecond laser generated grating in the core. The stress inducing tracks, BGWs, and FBGs were all written in the same scanning direction to avoid non-reciprocal "quill" effects [23].

\section{RESULTS}

Fig. 3 demonstrates the cumulative effect of increasing the waveguide birefringence, as shown by the widening of the polarization splitting in the FBG resonance with the addition of consecutive horizontally offsetted stress track. The $V$ and $H$ polarization modes were unresolved in this weak unstressed FBG, separated by $120 \mathrm{pm}$ with a single stress track and separated by $200 \mathrm{pm}$ with the addition of a second identical stress track on the opposite side of the waveguide. The horizontally offset stressing tracks increases the propagation constant of the $V$ polarization mode (shift to longer wavelengths in Fig. 3), leaving the $H$ mode of the same Bragg resonance largely unaffected.

The horizontal or vertical geometry of the parallel stressing tracks relative to the laser-writing direction was found previously to dramatically affect the induced birefringence [17]. Results are shown in Fig. 4 as a function of laser polarization (i.e., nanograting orientation), pulse energy of the stress inducing tracks, and geometric separation. These results are consistent with what has been demonstrated in the past [17], with new results obtained by HNA focusing and $100 \mathrm{kHz}$ repetition rate, shown here in the red curves.

The importance of the polarization of the writing laser and the separation from the stress inducing tracks to the waveguides was shown in this previous paper [17] and reproduced in Fig. 4 for the case of $500 \mathrm{kHz}$ repetition rate, LNA writing, with a $200 \mathrm{~nJ}$ pulse energy, and for both vertical and horizontal stress geometries. The benefit of the new results with $100 \mathrm{kHz}$ HNA focusing with a higher pulse energy of $\approx 300 \mathrm{~nJ}$ is then clearly demonstrated in Fig. 4 by a two-fold to three-fold overall in-

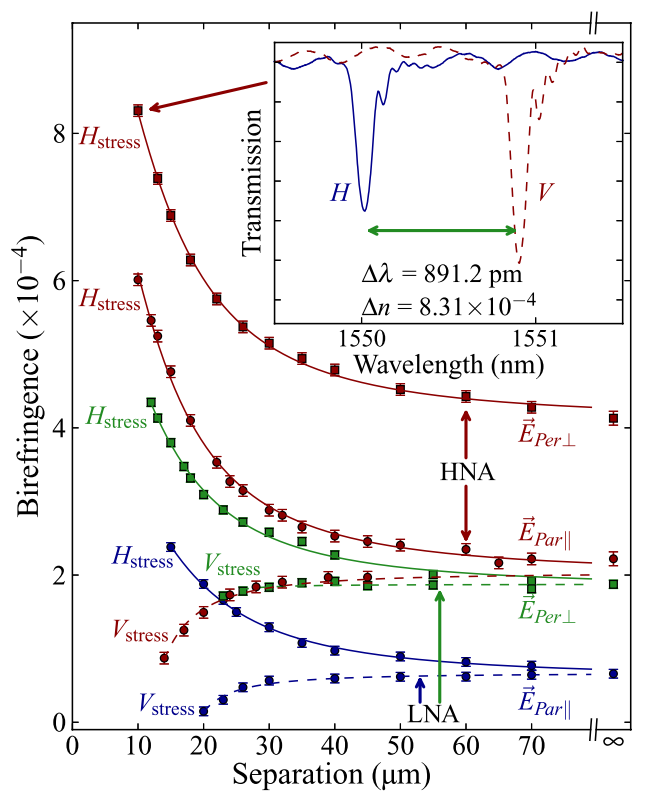

Fig. 4. Birefringence results as a function of stress tracks separation, $S$, for bulk fused silica waveguides with LNA (blue and green) [17] and HNA (red). The LNA results were fabricated with $500 \mathrm{kHz}$ repetition rate and $200 \mathrm{~nJ}$ pulse energy, while the the HNA results were produced with $100 \mathrm{kHz}$ repetition rate and $300 \mathrm{~nJ}$ pulse energy. Both focusing conditions are shown for parallel (circles) and perpendicular (squares) writing polarizations. The results for horizontal (solid lines -) and vertical (dashed line - -) geometries are shown for a selection of fabrication conditions. The inset shows an example spectrum, illustrating the birefringence measurement method comparing the transmission for both $V$ and $H$ polarization eigenmodes, for the highest birefringence value found in this set.

crease in the waveguide birefringence. The trends for vertical and horizontal stress, as well as the separation, are nearly identical as seen for the LNA cases. The inset of Fig. 4 shows the spectral separation of the $V$ and $H$ polarization modes for the case of the maximum stress in bulk glass achieved with the limit of highest pulse energy, and shortest spacing before the onset of evanescent coupling. The higher pulse energy available at such low repetition rates allowed for a much stronger impact of the stress created by the femtosecond laser generated tracks, resulting in a much larger birefringence with a maximum reported value of $\approx 8.3 \times 10^{-4}$ obtained with perpendicular polarization of the writing laser, stress tracks written with $\approx 300 \mathrm{~nJ}$, and separated by $10 \mu \mathrm{m}$ from the central BGW. However, reversing the sign of the birefringence still remains a challenge with the present approach.

The same method presented for bulk glass was then applied in SMFs in order to study the birefringence dependence on separation of the stress tracks for similar ranges of exposure and the same geometric orientations. Fig. 5 shows the results obtained with parallel polarization of the writing laser with different pulse energies and repetition rates applied in SMFs. Here, the pulse energy used for the stress tracks also has a significant impact on the amount of birefringence achieved. For the horizontal stress geometry and parallel polarization of the writing laser, a $\approx 5.65 \times 10^{-4}$ birefringence was found at $11 \mu \mathrm{m}$ of separation between the stress tracks and the fiber core, with $420 \mathrm{~nJ}$ of pulse energy and $100 \mathrm{kHz}$ of laser repetition rate. A birefringence 


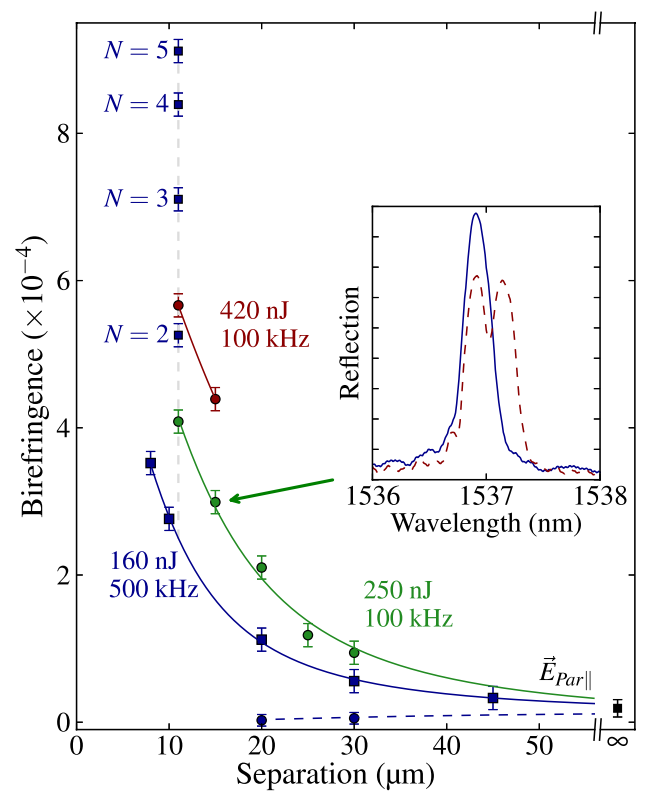

Fig. 5. Birefringence results as a function of stress tracks separation, $S$, for stress tracks in a SMF demonstrating similar behavior to the waveguides in fused silica shown in Fig. 4 for horizontal (solid lines -) and vertical (dashed line - -) geometries and written with parallel polarization $\left(\vec{E}_{\mathrm{Par} \|}\right)$ of the writing laser. The inset shows an example reflection spectrum of a FBG before (solid blue line -) and after (dashed red line - -) the fabrication of the stress tracks. The results for multiple stress tracks is shown in blue squares with $N=2,3,4$, and 5 representing the number of overwritten tracks for the case of $160 \mathrm{~nJ}$ pulse energy, further detailed in Fig. 6.

value below the limit of detection of this technique $\left(4 \times 10^{-6}\right)$ is found with vertical stress tracks at $20 \mu \mathrm{m}$ separation with $160 \mathrm{~nJ}$ of pulse energy and $500 \mathrm{kHz}$ of laser repetition rate. Results for perpendicular polarization stress tracks are not reported in Fig. 5, but are found to yield a similar increase as in the parallel polarization case.

As seen in Figs. 4 and 5, the data could be well represented by an inverse square function of the separation distance between the stress tracks and the BGWs (in Fig. 4) or the FBGs (in Fig. 5), with a horizontal asymptote at infinity equal to the reference birefringence value of the unstressed gratings under test.

These HNA focusing results in SMF and bulk glass are consistent with conclusions found previously for LNA stressing in bulk glass data [17], where the overall induction of birefringence in the waveguide is driven by stress generated by the laser modified regions and not the form birefringence within the stress track. On this basis, we further explore the possibility of building stronger birefringence in SMFs by adding more stress tracks around the waveguide or alternatively by overwriting the stress tracks already present.

Fig. 6 shows the birefringence recorded as a function of the number, $N$, of consecutive stress track pairs fabricated around the same FBG, following the geometry represented in the topleft illustration. Data are shown for both parallel (blue circles) and perpendicular (green squares) polarization of the femtosecond laser with an example spectrum shown in the bottom-right inset. The strongest birefringence of $2.0 \times 10^{-3}$ (red square) was obtained by combining multiple parallel tracks with per-

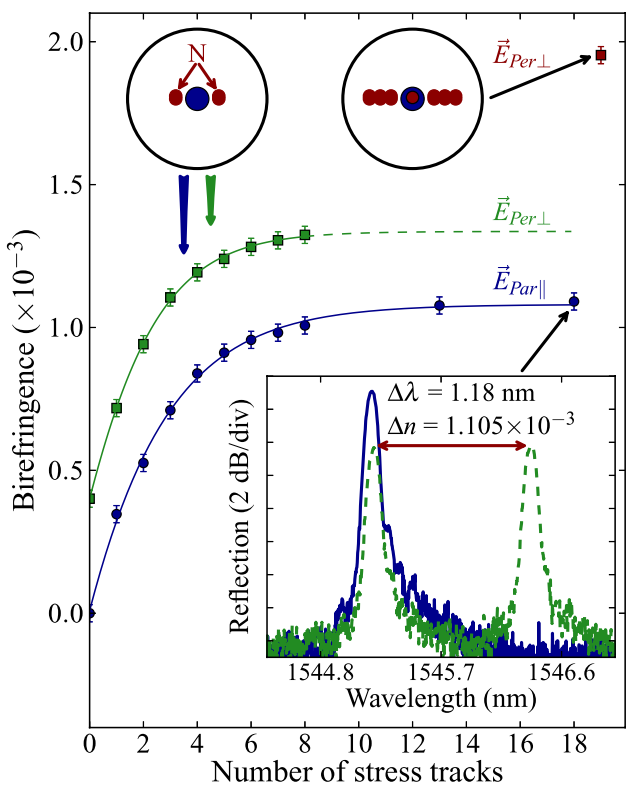

Fig. 6. Logistic-type saturation of the birefringence for multiple overwriting stress tracks placed $11 \mu \mathrm{m}$ away from the SMF core and fabricated with $\approx 160 \mathrm{~nJ}$ pulse energy at $500 \mathrm{kHz}$. The data and fitting are shown for parallel (blue circles and blue line) and perpendicular (green squares and green line) polarization of the writing laser. Inset: Example spectrum for 18 stress tracks at $11 \mu \mathrm{m}$ with parallel writing before (blue solid line -) and after (green dashed line - -) the fabrication of the stress tracks ensemble.

pendicular polarization forming a stressing plane (illustrated in the top-right inset of Fig. 6)

This benefit of increasing birefringence with overwriting of stressing tracks is clearly seen in Fig. 5 for the case of parallel horizontally stressing tracks, written with $160 \mathrm{~nJ}$ exposure, $11 \mu \mathrm{m}$ separation, and $N=2,3,4$, and 5 overwriting tracks. Such overwriting offers a large approximately three-fold increase in the birefringence of the SMF over the $N=1$ case, rising as a logistic-type function to a saturation level after $\approx 10$ scans as shown in Fig. 6. Here, parallel and perpendicular polarization writings yield similar enhancement at the same exposure conditions of $160 \mathrm{~nJ}$ pulse energy and $11 \mu \mathrm{m}$ separation, but with the result for perpendicular polarization showing an additional offset of $0.4 \times 10^{-3}$ birefringence, owing to the formation of strong birefringent nanogratings in the fiber core $(N=0)$ with the $90 \mathrm{~nJ}$ pulse energy used in the fabrication of the FBG. The $1.18 \mathrm{~nm}$ splitting of the FBG in the inset spectrum shows the strong $\Delta n=1.11 \times 10^{-3}$ response induced after full saturation with 18 overwritten stressing track pairs. These trends reinforce the previously reported observations [17] of the independent local and long reaching responses in the form and stress birefringence, respectively.

Given the maximum limit of overwriting on the present parallel tracks (see Fig. 6, top-left inset), a stronger birefringence was sought by writing multiple tracks horizontally to create lateral stressing planes (see Fig. 6 top-right inset) for a total of 19 tracks evenly distributed between $11 \mu \mathrm{m}$ and $8 \mu \mathrm{m}$ separation, symmetrically positioned on opposite sides of the SMF core, and fabricated with perpendicular polarization of the writing laser. The combination of strong stressing planes together with 


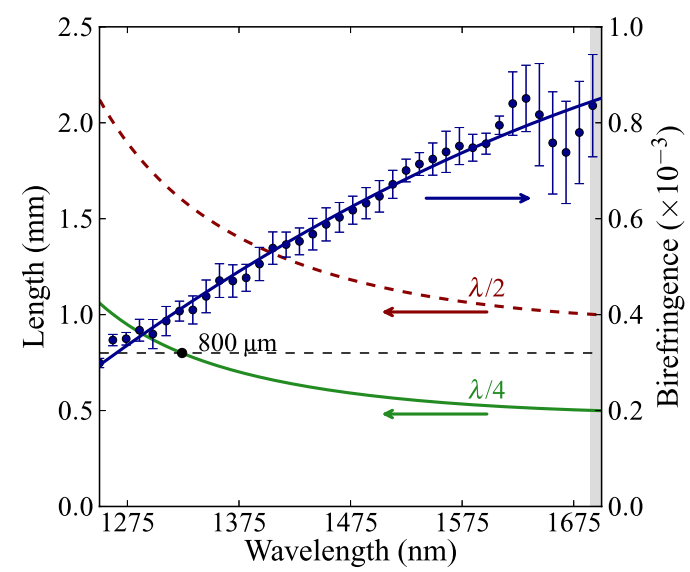

Fig. 7. Birefringence spectrum (blue circles) obtained from a cross polarization measurement made on a $800 \mu \mathrm{m}$ long (black horizontal dashed line) SMF segment with $N=10$ overlapping pairs of stress tracks written with parallel polarization in the horizontal geometry. The blue line is a polynomial fit to guide the eye. The length required for a zero-order half-wave plate (red dashed line - -) and a zero-order quarter-wave plate (green solid line -) is shown with the scale on the left, calculated based of the birefringence fit.

the form birefringence in the FBG core yielded a birefringence of $2.0 \times 10^{-3}$, exceeding by more than two-fold the previously reported maximum value found with a similar technique [15].

The birefringence values demonstrated here are very attractive for considering the design of very compact wave retarders of sub-millimeter lengths, in both bulk glass optical circuits or optical fibers, for applications in the infrared spectral band [4]. The possibility of extending in-line waveplates over a broad spectral band requires analysis of dispersion in the birefringence such as plotted over the $1250 \mathrm{~nm}$ to $1700 \mathrm{~nm}$ wavelength band in Fig. 7 (right scale). Here, the spectral birefringence, $\Delta n(\lambda)$, was determined by the cross polarization method [4] for the case of parallel polarization of the writing laser, with ten overwritten horizontal stress tracks $(500 \mathrm{kHz}$ laser repetition rate, $160 \mathrm{~nJ}$ pulse energy, and $11 \mu \mathrm{m}$ separation) forming a $800 \mu \mathrm{m}$ wave retarder in a SMF segment. These conditions produced a zero order retarder across the entire spectrum.

The minimum length, $L$, required for a quarter-wave or a halfwave retarder can be found from (1), where WP represents the wave retarder type $(\mathrm{WP}=1 / 2$ for a half-wave and $\mathrm{WP}=1 / 4$ for a quarter-wave plate):

$$
L=\mathrm{WP} \frac{\lambda}{\Delta n}
$$

This results in very short devices of $0.5 \mathrm{~mm}$ to $2 \mathrm{~mm}$ lengths for the quarter-wave and half-wave plates as shown by the calculated results in Fig. 7 (left scale) over a large spectral range from $1250 \mathrm{~nm}$ to $1700 \mathrm{~nm}$. In the particular case of the $800 \mu \mathrm{m}$ long device used for this measurement, a quarter-wave plate was found at $1324 \mathrm{~nm}$ wavelength.

The design of broadband waveplates requires the length, $L$, to be constant in (1) over a large wavelength range. The condition for constant retardance is satisfied by (2) when the relative

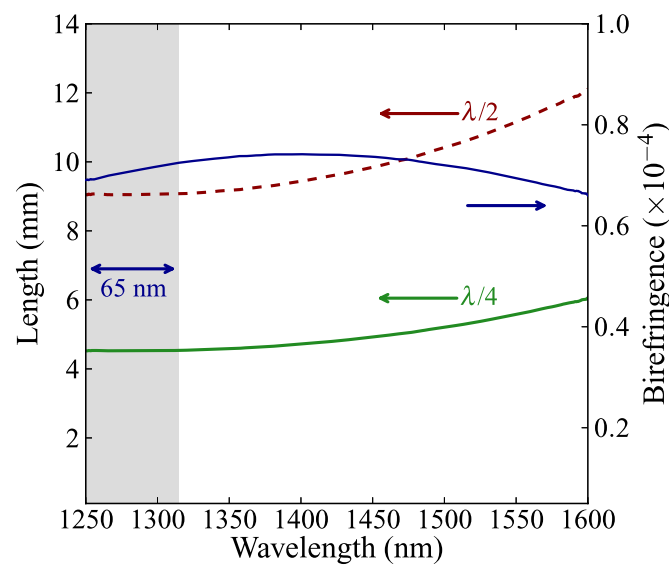

Fig. 8. Waveguide length (left scale) required for zero-order quarter-wave and zero-order half-wave retarders as a function of wavelength, for a waveguide written with $110 \mathrm{~nJ}$ pulse energy in bulk fused silica with parallel laser polarization. The birefringence spectrum (blue line) follows the right scale. A $65 \mathrm{~nm}$ bandwidth (gray shaded area) offers a constant retardance where broadband waveplates are available [4].

variation of birefringence is the same as the relative variation of wavelength:

$$
\frac{d(\Delta n)}{\Delta n}=\frac{d \lambda}{\lambda}
$$

This manifests in a $10 \mathrm{~nm}$ band of constant retardance between $1690 \mathrm{~nm}$ and $1700 \mathrm{~nm}$ in the specific case of Fig. 7, defined within a length variation tolerance of $0.2 \%$. An extrapolation of the shallow slope suggests a much broader band is available for this waveplate toward longer wavelength. Alternatively, further balancing of the femtosecond laser exposure between the SMF core and cladding could preferentially tune the birefringence dispersion to better meet the broadband condition of (2) in other spectral windows.

The formation of broadband waveplates is also available in waveguides formed in bulk fused silica. Following the case of $110 \mathrm{~nJ}$ laser exposure of horizontal stressing tracks, the birefringence dispersion reported in the past (see [4, Fig. 4]) is shown to meet the broadband condition of (2) across the $1250 \mathrm{~nm}$ and $1315 \mathrm{~nm}$ range as shown in the plot of the minimum waveplate length in Fig. 8. The expected retardance variation is less than $\pm 0.005 \mathrm{rad}$ in this spectral range, equivalent to $\mathrm{a} \approx 0.2 \%$ of tolerance in the length. Hence, laser exposure conditions can be varied independently in forming, or directly modifying, the waveguide by controlling the pulse energy, writing speed, and polarization of the writing laser, and in controlling the stress generated by the surrounding laser tracks.

\section{Discussion}

In SMFs, weak and strong FBGs were initially generated to vary the local waveguide birefringence in a moderate range of $\approx 10^{-7}$ to $4 \times 10^{-4}$, owing to the polarization controlled orientation of laser generated nanogratings. The addition of surrounding stressing tracks further increased the birefringence in 
a highly controllable way to values as large as $2 \times 10^{-3}$ (see Fig. 5), following exposure tuning trends similar to those reported previously in bulk fused silica (see Fig. 4). Namely, the vertical geometry of the stressing lines only weakly opposed any birefringence first induced during the FBG fabrication, while the horizontal geometry strongly increased this birefringence, rising further for higher pulse energy and closer spacing (see Figs. 4 and 5), and also with overwriting stress inducing laser tracks (see Fig. 6). The birefringence values presented here for laser stressed SMFs are high in comparison with commercial polarization maintaining fibers that have typical beat length ranging from $1.5 \mathrm{~mm}$ to $5 \mathrm{~mm}$. One expects a short beat length of $775 \mu \mathrm{m}$ for the presently available birefringence of $2 \times 10^{-3}$ at $1550 \mathrm{~nm}$.

The laser overwriting approach generated strong and tunable birefringence (see Fig. 6) that offers a means for precise in situ tuning of the waveguide birefringence that is attractive where high accuracy phase control is necessary, such as designing waveplates for specific wavelengths. Higher stress fields could be designed into waveguide devices by a combination of overwritten stress tracks with varying scan speeds to further increase the net fluence. In fibers, when higher losses can be tolerated, perpendicular polarization of the writing laser and high pulse energies are preferred to generate form birefringence values comparable to what is found in bulk fused silica, further adding to the stress birefringence.

A design for a fiber-optic polarimetric twist and torsion sensors was recently demonstrated with polarization maintaining fibers spliced to an SMF [11]. The birefringent sections required for this type of sensor could be made considerably smaller by the technique presented in this paper. Shorter wave retarders could enable in-line polarization devices such as quarter-wave absorbers for fibers laser or polarization converters that can be fabricated directly in optical fiber, eliminating the need for bulk, free space components, or spliced fiber elements.

The high birefringence levels found here may have an impact on the design of polarization dependent devices such as polarization beam splitters [5] in both bulk glass and optical fiber platforms. More compact planar light circuits or in-fiber devices can lead to higher integration of polarization encoded quantum optics measurements in smaller volumes and with shorter waveguide segments.

\section{CONCLUSION}

The results presented here expand on a previously demonstrated technique [15], [17] to tune the waveguide birefringence with femtosecond laser writing of adjacent stress tracks. The technique was extended to SMFs with oil immersion laser writing to overcome optical aberrations. The birefringence tuning range was greatly enhanced, compared to values reported in the past, by optimization of the exposures and structural geometry. The use of tighter focusing condition with an HNA oil immersion lens, higher pulse energy, and multiple overwriting stress enabled a maximum birefringence saturation value of $2 \times 10^{-3}$, demonstrating a 2.5 -fold increase over the previous maximum reported with femtosecond laser radiation.

\section{REFERENCES}

[1] V. R. Bhardwaj, P. B. Corkum, D. M. Rayner, C. Hnatovsky, E. Simova, and R. S. Taylor, "Stress in femtosecond-laser-written waveguides in fused silica," Opt. Lett., vol. 29, no. 12, pp. 1312-1314, 2004.

[2] W. Yang, E. Bricchi, P. G. Kazansky, J. Bovatsek, and A. Y. Arai, "Self-assembled periodic sub-wavelength structures by femtosecond laser direct writing," Opt. Exp., vol. 14, no. 21, pp. 10117-10124, 2006

[3] Z. Liu, C. Wu, M.-L. V. Tse, C. Lu, and H.-Y. Tam, "Ultrahigh birefringence index-guiding photonic crystal fiber and its application for pressure and temperature discrimination," Opt. Lett., vol. 38, no. 9, pp. 1385-1387, 2013.

[4] L. A. Fernandes, J. R. Grenier, P. R. Herman, J. S. Aitchison, and P. V. S. Marques, "Femtosecond laser writing of waveguide retarders in fused silica for polarization control in optical circuits," Opt. Exp., vol. 19, no. 19, pp. 18294-18301, 2011.

[5] L. A. Fernandes, J. R. Grenier, P. R. Herman, J. S. Aitchison, and P. V. S. Marques, "Femtosecond laser fabrication of birefringent directional couplers as polarization beam splitters in fused silica," Opt. Exp., vol. 19, no. 13, pp. 11992-11999, 2011.

[6] S. Betti, G. De Marchis, and E. Iannone, "Polarization modulated direct detection optical transmission systems," J. Lightw. Technol., vol. 10, no. 12, pp. 1985-1997, Dec. 1992.

[7] J. L. O'Brien, A. Furusawa, and J. Vuckovic, "Photonic quantum technologies," Nat. Photon., vol. 3, no. 12, pp. 687-695, 2009.

[8] M. Lobino and J. L. O'Brien, "Entangled photons on a chip," Nature, vol. 469, no. 7328, pp. 43-44, 2011.

[9] T. Geernaert, T. Nasilowski, K. Chah, M. Szpulak, J. Olszewski, G. Statkiewicz, J. Wojcik, K. Poturaj, W. Urbanczyk, M. Becker, M. Rothhardt, H. Bartelt, H. Berghmans, and H. Thienpont, "Fiber Bragg gratings in germanium-doped highly birefringent microstructured optical fibers," IEEE Photon. Technol. Lett., vol. 20, no. 8, pp. 554-556, Apr. 2008

[10] O. Frazao, C. Jesus, J. M. Baptista, J. L. Santos, and P. Roy, "Fiberoptic interferometric torsion sensor based on a two-LP-mode operation in birefringent fiber," IEEE Photon. Technol. Lett., vol. 21, no. 17, pp. 1277 1279, Sep. 2009.

[11] D. Lesnik and D. Donlagic, "In-line, fiber-optic polarimetric twist/torsion sensor.," Opt. Lett., vol. 38, no. 9, pp. 1494-1496, 2013.

[12] N. Jovanovic, J. Thomas, R. J. Williams, M. J. Steel, G. D. Marshall, A. Fuerbach, S. Nolte, A. Tünnermann, and M. J. Withford, "Polarizationdependent effects in point-by-point fiber Bragg gratings enable simple, linearly polarized fiber lasers," Opt. Exp., vol. 17, no. 8, pp. 6082-6095, 2009.

[13] R. Goto, R. J. Williams, N. Jovanovic, G. D. Marshall, M. J. Withford, and S. D. Jackson, "Linearly polarized fiber laser using a point-by-point Bragg grating in a single-polarization photonic bandgap fiber.," Opt. Lett., vol. 36, no. 10, pp. 1872-1874, 2011.

[14] C. C. Willis, E. McKee, P. Böswetter, A. Sincore, J. Thomas, C. Voigtländer, R. G. Krämer, J. D. Bradford, L. Shah, S. Nolte, A. Tünnermann, and M. Richardson, "Highly polarized all-fiber thulium laser with femtosecond-laser-written fiber Bragg gratings," Opt. Exp., vol. 21, no. 9, pp. 10467-10474, 2013.

[15] D. Grobnic, S. J. Mihailov, and C. W. Smelser, "Localized high birefringence induced in SMF-28 fiber by femtosecond IR laser exposure of the cladding," J. Lightw. Technol., vol. 25, no. 8, pp. 1996-2001, Aug. 2007.

[16] K. Chah, D. Kinet, M. Wuilpart, P. Mégret, and C. Caucheteur, "Femtosecond-laser-induced highly birefringent Bragg gratings in standard optical fiber," Opt. Lett., vol. 38, no. 4, pp. 594-596, 2013.

[17] L. A. Fernandes, J. R. Grenier, P. R. Herman, J. S. Aitchison, and P. V. S. Marques, "Stress induced birefringence tuning in femtosecond laser fabricated waveguides in fused silica," Opt. Exp., vol. 20, no. 22, pp. 24 103-24114, 2012

[18] J. Thomas, C. Voigtländer, R. Becker, D. Richter, A. Tünnermann, and S. Nolte, "Femtosecond pulse written fiber gratings: A new avenue to integrated fiber technology," Laser Photon. Rev., vol. 6, no. 6, pp. 709723, 2012.

[19] J. R. Grenier, L. A. Fernandes, and P. R. Herman, "Femtosecond laser writing of optical edge filters in fused silica optical waveguides," Opt. Exp., vol. 21, no. 4, pp. 4493-4502, 2013

[20] H. Zhang, S. M. Eaton, and P. R. Herman, "Single-step writing of Bragg grating waveguides in fused silica with an externally modulated femtosecond fiber laser," Opt. Lett., vol. 32, no. 17, pp. 2559-2561, 2007. 
[21] L. A. Fernandes, J. R. Grenier, P. R. Herman, J. S. Aitchison, and P. V. S. Marques, "Femtosecond laser writing of polarization devices for optical circuits in glass," Proc. SPIE, vol. 8247, pp. 82470M-9, 2012.

[22] L. Ramirez, M. Heinrich, S. Richter, F. Dreisow, R. Keil, A. V. Korovin, U. Peschel, S. Nolte, and A. Tünnermann, "Tuning the structural properties of femtosecond-laser-induced nanogratings," Appl. Phys. A, vol. 100, no. 1, pp. 1-6, 2010.

[23] W. Yang, P. G. Kazansky, and Y. P. Svirko, "Non-reciprocal ultrafast laser writing," Nat. Photon., vol. 2, no. 2, pp. 99-104, 2008.

Luís A. Fernandes received the degree in applied physics and the Ph.D. degree in physics from the Faculty of Science, University of Porto, Porto, Portugal, in 2006 and 2012, respectively. In 2008, he was awarded a Ph.D. fellowship from the Fundação para a Ciência e Tecnologia for his thesis entitled "Birefringence and Bragg grating control in femtosecond laser written optical circuits" researched in collaboration with the Photonics Group at the University of Toronto, ON, Canada, where he became a Postdoctoral Fellow in 2013. He was awarded a SPIE Scholarship in Optical Science and Engineering and an award for best oral presentations in 2010. He served as member of the board of the University of Toronto SPIE Student Chapter from 2010 to 2013 and is a member of the SPOF, SPF, SPIE, OSA, and IEEE Photonics Society.

Jason R. Grenier received the B.A.Sc. and M.A.Sc. degrees in electrical engineering from the University of Waterloo, Waterloo, ON, Canada, in 2003 and 2006, respectively. As a Ph.D. candidate in the Department of Electrical and Computer Engineering, the University of Toronto, his research involves using femtosecond lasers to expand the frontiers of three-dimensional fabrication of novel optical devices in the cladding of optical fibers. He has held an NSERC post-graduate and an Ontario Graduate Scholarship, has received several best paper awards, and has recently won awards from the SPIE, the Canadian Institute for Photonics Innovation, and the Ontario Centres of Excellence. He is the founding President of the University of Toronto SPIE Student Chapter and is also an OSA member.
Paulo V. S. Marques received the Ph.D. degree in physics from the University of Porto, Porto, Portugal, in 2000. He is currently an Auxiliary Professor at the Department of Physics and Astronomy, Faculty of Science, University of Porto. $\mathrm{He}$ is a Researcher and current Unit Coordinator at the Optoelectronics and Electronic Systems Unit (UOSE) of INESC-TEC. His research interests include integrated optics and microfabrication, integrated sensors, and laser direct writing of optical devices. He has published more than 100 scientific journal papers and international conference proceedings, and holds four worldwide patents.

J. Stewart Aitchison received the B.Sc. degree (First Class Hons.) in 1984 and the Ph.D. degree in 1987 from the Department of Physics, Heriot-Watt University, Edinburgh, U.K. From 1988 to 1990, he was a Postdoctoral Member of Technical Staff at Bellcore. He joined the ECE Department at the University of Glasgow in 1990 and became Professor of Photonics in 1999. In 1996, he was the holder of a Royal Society of Edinburgh Personal Fellowship and carried out research on spatial solitons as a Visiting Researcher at CREOL, University of Central Florida. Since 2001, he has been a Professor in the ECE Departmen at the University of Toronto, ON, Canada. He is a Fellow of the Royal Society of Canada, the IOP, and the OSA.

Peter R. Herman received the M.A.Sc. and Ph.D. degrees studying lasers and diatomic spectroscopy in the Department of Phsyics, University of Toronto, ON, Canada, in 1982 and 1986, respectively. Following the postdoctoral study of X-ray lasers at the Institute of Laser Engineering in Osaka University, he joined the ECE Department at the University of Toronto where he holds a Ful Professor position. He guides a large research group that develops and applies laser technology and advanced beam delivery systems to control and harvest laser interactions in new frontiers of 3-D nanofabrication. His group interacts with numerous academic and industrial partners. He has published more than 250 scientific journals and conference papers. He is a Fellow of the Optical Society of America, and a member of the SPIE. 